
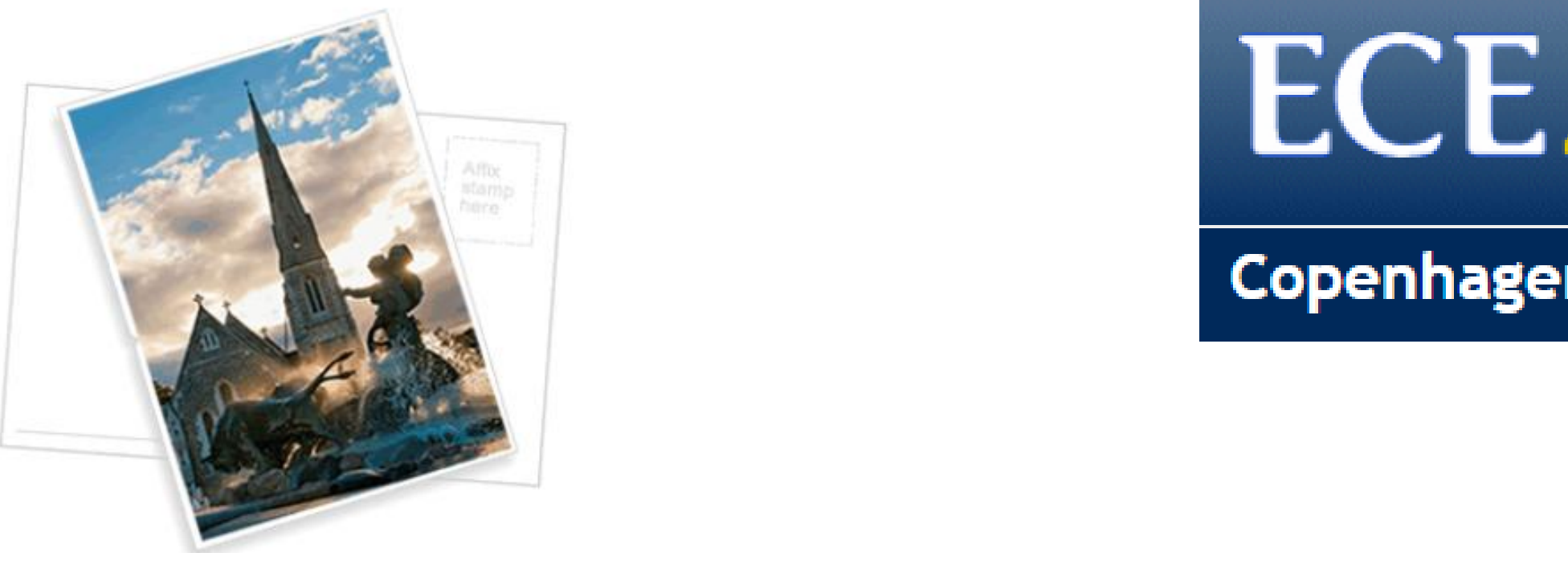

\title{
Copenhagen, Denmark
}

\section{Subacute thyroiditis: unusual}

\section{presentation and diagnostic troubles}

Rosa Maria Paragliola, Maria Pia Ricciato, Vincenzo Di Donna, Laura Castellino, Rosa Maria Lovicu, Alfredo Pontecorvi, Salvatore M. Corsello

Endocrinology Unit, Catholic University School of Medicine, Rome 


\section{Introduction}

Subacute thyroiditis is a spontaneously remitting inflammatory condition of thyroid gland.

The disease is characterized by a whole gland involvement while thyroidal radioactive iodine uptake is depressed.

Laboratory evaluation during the early acute phase usually shows:

transient thyrotoxicosis

* elevation of serum thyroglobulin concentration

* elevation of erythrocyte sedimentation rate

The most important symptom is severe pain and extreme tenderness in the thyroid region. 


\section{Case report}

A 73 years old man came to our observation for severe dysphagia and loss of weight (10 kg in one month). One week before the first medical evaluation, he suspended all drugs per os because he could not swallow pills and food.

About 30 years before he underwent coronary artery bypass graft because of myocardial infarction.

Thyroid function test were performed, showing a severe hyperthyroidism:

FT4 $40 \mathrm{pg} / \mathrm{ml}$
FT3 $11.9 \mathrm{pg} / \mathrm{ml}$
TSH $<0.01 \mathrm{mcU} / \mathrm{ml}$
Anti TSH receptor antibodies: $0.1 \mathrm{U} / \mathrm{L}(<1.75)$ Anti TPO anti antibodies: $2.09 \mathrm{UI} / \mathrm{ml}(<4.10)$ Anti Tg antibodies: $<0.5 \mathrm{Ul} / \mathrm{ml}(<5.6)$

Thyroid ultrasound showed an increased gland with inhomogeneous pattern, without nodules or abnormal vascularization.

Patient did not take amiodarone 


\section{Case report}

Patient started methimazole $30 \mathrm{mg} /$ die, without any benefits.

Patient came to our evaluation about one month after the onset of symptoms.

Clinical evaluation showed tachycardia, enlarged and tender thyroid gland at neck palpation without relevant pain.

* Biochemical evaluation showed increased VES and C-reactive protein.

* Thyroid scintigraphy was not performed because of the possible interference caused by iodinate contrast medium (coronary angiography few days before).

Neverthless subacute thyroiditis was strongly probable 


\section{Case report}

Therefore, patient started steroid therapy:

i.v. methylprednisolone 40 for about two weeks and then prednisone $25 \mathrm{mg} /$ day, which was tapered and continued for 30 days.

\section{Methimazole was stopped}

Clinical symptoms, and in particular dysphagia, improved after few days of i.v. methylprednisolone while biochemical evaluation performed after two months showed a normalization of thyroid function test and inflammatory parameters.

\section{ECE2013}




\section{Conclusion}

We here described an unusual case of subacute thyroidits in which only dysphagia and thyrotoxicosis, without anterior neck pain, suggested an inflammatory condition.

Diagnosis was made on the basis of clinical and laboratory features, because thyroid scintigraphy, which can be resolutive for differential diagnosis in uncertain condition, was not possible to be performed. 